CERN-PH-TH/2005-080

IFUP-TH/2005-12

UCB-PTH-05/15

LBNL-57568

\title{
Dark Energy and Right-Handed Neutrinos
}

\author{
Riccardo Barbieri $^{a, b}$, Lawrence J. Hall ${ }^{c}$, Steven J. Oliver ${ }^{c}$, Alessandro Strumia $^{d}$ \\ a Scuola Normale Superiore and INFN, Piazza dei Cavalieri 7, I-56126 Pisa, Italy \\ ${ }^{b}$ Theoretical Physics Division, CERN, CH-1211 Genève 23, Switzerland \\ c Department of Physics, University of California, Berkeley, and \\ Theoretical Physics Group, LBNL, Berkeley, CA 94720, USA \\ d Dipartimento di Fisica dell'Università di Pisa and INFN, Italia
}

\begin{abstract}
We explore the possibility that a $\mathrm{CP}$ violating phase of the neutrino mass matrix is promoted to a pseudo-Goldstone-boson field and is identified as the quintessence field for Dark Energy. By requiring that the quintessence potential be calculable from a Lagrangian, and that the extreme flatness of the potential be stable under radiative corrections, we are led to an essentially unique model. Lepton number is violated only by Majorana masses of light right-handed neutrinos, comparable to the Dirac masses that mix right- with left-handed neutrinos. We outline the rich and constrained neutrino phenomenology that results from this proposal.
\end{abstract}




\section{Introduction}

A series of different observations and considerations [1, 2] provides a strong case for a striking phenomenon: the expansion of the universe has recently begun to accelerate. Although a definitive experiment with sufficiently small systematic uncertainties is lacking, if confirmed this remarkable fact calls for an adequate explanation and, even more important, motivates the search for other correlated observable phenomena.

The accelerated expansion of the universe could be due to a tiny Cosmological Constant (CC), $\Lambda \approx\left(3 \cdot 10^{-3} \mathrm{eV}\right)^{4}$; tiny, but non-zero. In fact, the frustration generated by the unsuccessful attempts to solve the vacuum energy problem has led to the development of a mild anthropic interpretation of the apparently observed value of the $\mathrm{CC}$ [3]. Here we take the view that the search for a more fundamental understanding of the cosmic acceleration remains highly motivated, even if still resting on the assumption of an exactly vanishing vacuum energy.

As an alternative to a CC, the accelerated expansion of the universe may be due to the evolution of some scalar field, uniform or quasi-uniform in space, with the associated "Dark Energy" (DE) mostly in its potential, usually called "quintessence" [4. Signals related to such an interpretation, although typically only vaguely determined, could be an equation of state of the associated fluid different from the one of a pure $\mathrm{CC}$, or the effects of the couplings of the quintessence field to the usual matter or gauge fields.

In this work we describe a possible microscopic origin for the quintessence field and for its potential, guided by two general requirements and one phenomenological observation. One general requirement is that the quintessence potential should be calculable from a Lagrangian and its peculiar properties, in particular its extreme flatness, should be stable under radiative corrections. Since the mass scale governing this flatness is todays Hubble parameter, $H_{0} \approx 10^{-33} \mathrm{eV}$, this requirement is severe indeed, although it is known to be satisfied by a Pseudo-Goldstone Boson (PGB) arising from the spontaneous breaking of a global symmetry near the Planck scale [5]. Our second general requirement is that the physics of DE be directly connected to observable particle physics, so that the resulting theory can be tested in the laboratory. These two requirements appear to conflict - if the quintessence field is coupled sufficiently strongly to the standard model to give laboratory signals, then radiative corrections involving this coupling will destroy the extreme flatness of the potential. A hint of a possible escape from this conundrum is provided by the phenomenological observation, already made by several people [6, 7], of the relative closeness of the energy scale associated with DE to the scale of neutrino masses. Thus we are led to explore the possibility that a $\mathrm{CP}$ violating phase of the neutrino mass matrix is promoted to a PGB field and is identified as the quintessence field for DE. 


\section{The model}

To implement this idea we introduce right-handed neutrinos, $N_{i}$, at least two but most likely three, and as many complex scalars, $\phi_{i j}$, as there are independent Lorentz-invariant neutrino bilinears $N_{i} N_{j}$. These scalars have Yukawa couplings to the $N_{i}\left(\phi_{i j}=\phi_{j i}, \lambda_{i j}=\lambda_{j i}\right)$

$$
\mathscr{L}_{Y}^{N}=\frac{1}{2} \sum_{i j} \lambda_{i j} \phi_{i j} N_{i} N_{j},
$$

which are invariant under independent phase transformations of each $N_{i}$ field, say $\mathrm{U}(1)^{3}$ for concreteness. $\mathrm{U}(1)^{3}$ is a subgroup of the $\mathrm{U}(1)^{6}$ which transforms each of the $\phi_{i j}$-fields by an independent phase. The crucial assumption is that, in the limit of vanishing $\lambda_{i j}$, the full Lagrangian is invariant under this global $\mathrm{U}(1)^{6}$, which is spontaneously broken by the vacuum expectation values $\left\langle\phi_{i j}\right\rangle \equiv f_{i j}$.

In the absence of any other coupling of the $N_{i}$ fields, this model has three massless Goldstone bosons and three PGBs, $G_{i j}$. The effective potential for the combinations of $G_{i j}$ that are PGBs arises at one loop and is given by

$$
V_{1} \approx \frac{1}{32 \pi^{2}} \operatorname{Tr}\left[M M^{\dagger} M M^{\dagger} \ln \frac{\Lambda^{2}}{M M^{\dagger}}\right]
$$

where $M_{i j}=\lambda_{i j} f_{i j} e^{i G_{i j} / f_{i j}}$ is the field-dependent, right-handed neutrino mass matrix and $\Lambda$ is a cut-off, to be specified later. Note the irrelevance in $V$ of any quadratic term in $M_{i j}$, however generated, since the only such term invariant under $\mathrm{U}(1)^{3}$ is also $\mathrm{U}(1)^{6}$-invariant, and therefore $G_{i j}$ independent. A typical term in $V_{1}$ contributing to the potential of a PGB field, $G$, has the form

$$
V(G)=\mu^{4} \cos (G / f)
$$

where $\mu^{4}=O\left(M^{4}\right)$ arises as a product $M_{i j} M_{j k}^{*} M_{k l} M_{l i}^{*}$, and $f$ is an appropriate function of the symmetry breaking parameters $f_{i j}$. It is well-known that, with $\mu \approx 3 \times 10^{-3} \mathrm{eV}$ and $f$ of order $M_{\mathrm{Pl}}, G$ is a consistent candidate for the quintessence field [5, 6, 8]. However, the signals we wish to stress are not those that come from the form of the potential (3), but rather are due to the required form for the underlying neutrino sector.

Two natural and important questions arise at this point. Could we interpret the $N_{i}$ as the lefthanded neutrinos entering the usual left-handed lepton doublets $L_{i}$ ? What other couplings can complete consistently the neutrino sector? To answer the first question we should first transform eq. (11) into a gauge invariant interaction involving the $L_{i}$ and the Higgs doublet $h$

$$
\mathscr{L}_{Y}^{L}=\frac{1}{2} \sum_{i j} \lambda_{i j}^{L} \phi_{i j} \frac{h^{2}}{M_{L}^{2}} L_{i} L_{j},
$$

where gauge indices are left understood and $M_{L}$ is an energy scale introduced to give $\mathscr{L}_{Y}^{L}$ the correct dimensions. Indeed, if we now replace the Higgs field with its vacuum expectation value, 
we would be led to the same contribution as in (2) to the PGB potential, except that $M_{i j}$ would now be the left-handed neutrino mass matrix. Apparently this is the minimal theory, with the DE field directly related to the phases of the $3 \times 3$ neutrino mixing matrix. It is straightforward to see, however, that radiative corrections above the weak scale with internal Higgs fields destroy radiative stability. This is an important conclusion. Without considerable complications of the Higgs sector 9], the introduction of light right-handed neutrinos appears as a necessity. A similar conclusion applies to the case with $\phi_{i j}$ fields coupled to the $L_{i} N_{j}$ bilinear.

We can now answer the second question stated above. A consistent completion of the Yukawa Lagrangian in the lepton sector has the form

$$
\mathscr{L}_{Y}=\sum_{i j} \lambda_{i j}^{E} h L_{i} e_{j}^{c}+\sum_{i j} \lambda_{i j}^{D} h L_{i} N_{j}+\frac{1}{2} \sum_{i j} \lambda_{i j} \phi_{i j} N_{i} N_{j}
$$

involving the right-handed charged leptons, $e_{i}^{c}$. One recognizes the usual Dirac neutrino mass matrix, proportional to $\lambda^{D}$, and one notices the absence of any (gauge invariant) Majorana mass term for the left-handed neutrinos of the form $\lambda_{i j}^{M} h^{2} L_{i} L_{j} / M_{L}$. Such a term, in fact, would explicitly break lepton number and, in conjunction with the Dirac mass matrix, would allow terms in the PGB potential linear in $M_{i j}$, thus also destroying radiative stability. In fact, radiative stability requires that all non-renormalizable operators conserve both $\mathrm{U}(1)^{6}$ on the $\phi_{i j}$ and an overall lepton number, $\mathrm{U}(1)_{L}$. In the presence of non-zero $\lambda_{i j}$, as well as generic $\lambda^{E, D}$ couplings, the $\mathrm{U}(1)^{6}$ symmetry is explicitly broken to $\mathrm{U}(1)_{L}$; it is convenient to label the theory by "U(1) $\rightarrow$ $\mathrm{U}(1)_{L} "$.

From the above arguments, the theory of (5) is essentially unique. The only fermion bilinear that $\phi_{i j}$ can couple to is $N_{i} N_{j}$, and the $h^{2} L_{i} L_{j}$ operator must be absent. We conclude that there must be two or more light right-handed neutrinos, with typical entries in their Majorana mass matrix of order $3 \times 10^{-3} \mathrm{eV}$ - broadly comparable to the entries in the Dirac matrix. It is remarkable that, in promoting a $\mathrm{CP}$ violating phase of the neutino sector to a field, the mass of this field can be protected to the level of $H_{0} \approx 10^{-33} \mathrm{eV}$. The key is to ensure that the leading radiative correction to $m_{G}^{2}$ is of order $m_{\nu}^{4} / M_{\mathrm{Pl}}^{2}$.

It is the Dirac mass term in (5) that allows us to call $N_{i}$ the right-handed neutrinos. One may wonder whether this term introduces significant new corrections to the PGB potential. In fact it does at two-loop order, giving a term in the PGB potential

$$
V_{2} \approx\left(\frac{1}{16 \pi^{2}}\right)^{2} \operatorname{Tr}\left(M M^{\dagger} \lambda^{D} \lambda^{D \dagger}\right) \Lambda^{2} .
$$

This leads us to consider a supersymmetric extension of the model with spartner masses at the Fermi scale, in which case a typical sneutrino mass cuts off both the quadratic divergence of this two-loop potential and the logarithmic divergence of the one-loop term in (2). Up to loop factors, the resulting two-loop contribution also becomes of the relevant order of magnitude for 
a quintessence potential ${ }^{1}$. Note that with the complete $\mathscr{L}_{Y}$ all the would-be-Goldstones associated with the breaking of $\mathrm{U}(1)^{6}$ acquire a mass from the PGB potential, except for the linear combination related to the overall lepton number.

We must be clear that we have not explained why the neutrinos are light - quite the reverse, since we have introduced extremely small parameters in the $\lambda$ and $\lambda^{D}$ matrices. The PGB masses are small because they are proportional to powers of these small explicit symmetry breaking parameters. Neglecting flavor labels and emphasizing only the very small parameters, the interactions of (5) can be rewritten as $h L e^{c}+\varepsilon_{D} h L N+\varepsilon_{M}^{2} \phi N N$, where $\varepsilon_{D, M}$ are now the small parameters. These parameters may not be promoted to fields, with the lightness of the neutrinos explained in terms of a small vacuum value, since these fields would lead to disastrous radiative corrections to the PGB potential. Nevertheless, it is interesting that both $\varepsilon_{D}$ and $\varepsilon_{M}$ should take on values of order $10^{-13}$ to $10^{-15}$ for acceptable neutrino masses and DE - an approximate symmetry acts on the $N$ fields. In higher dimensional theories, a small $\varepsilon$ could result if $N$ propagate in a bulk and have a small, exponentially suppressed wavefunction at the location of the $\phi, L$ and $h$ fields. Even in this case, any parameter that sets the geometrical configuration must not correspond to a light field in the low energy effective four dimensional theory.

Two variations in the theory are possible: by restricting the form of the couplings or the number of $\phi$ fields in (5), alternative symmetry breaking patterns emerge, yielding PGB potentials different from $V_{1}+V_{2}$ of the generic case. If the entire theory possesses an exact $\mathrm{U}(1)_{i}^{3}$ symmetry, with one $\mathrm{U}(1)$ for each lepton generation, $\lambda^{E, D}$ become diagonal and we obtain the "U(1) ${ }^{6} \rightarrow \mathrm{U}(1)_{i}^{3}$ " variation. Since $\operatorname{Tr}\left(M M^{\dagger} \lambda^{D} \lambda^{D^{\dagger}}\right)$ is now independent of the three PGB fields, the potential for the PGBs is given purely by $V_{1}$ of (2). As this potential has only a logarithmic divergence, the quintessence potential is stable to radiative corrections whether or not superpartners are at the weak scale. In this variation, lepton flavor mixing arises entirely from spontaneous breaking. Finally, if the initial symmetry is restricted to $\mathrm{U}(1)_{i}^{3}$, so that the theory possesses only three $\phi$ fields, $\phi_{i i}$, we obtain the "U(1) ${ }_{i}^{3} \rightarrow \mathrm{U}(1)_{L}$ " variation. The potential for the 2 PGBs occurs at 3 loops

$$
V_{3} \approx\left(\frac{1}{16 \pi^{2}}\right)^{3} \operatorname{Tr}\left(M \lambda^{D \dagger} \lambda^{D} M^{\dagger} \lambda^{D} \lambda^{D \dagger}\right) \Lambda^{2} .
$$

This again gives a successful theory for quintessence with entries of $M$ of order $10^{-3} \mathrm{eV}$, but, in contrast to the general case, supersymmetry should be absent, giving a cutoff $\Lambda \approx M_{\mathrm{Pl}}$. As the cutoff is reduced, so the entries of $M$ can be made larger — with the cutoff in this variation at the weak scale the right-handed neutrino masses may be raised to an $\mathrm{MeV}$.

\footnotetext{
${ }^{1}$ In contrast to the case of Mass Varying Neutrinos [7, the effective contribution to the quintessence potential from the cosmological neutrino density is numerically irrelevant. We can also ignore the small variation of the neutrino masses induced by their dependence on the dynamical PGB fields.
} 


\section{Constraints and Neutrino Spectra}

Since $f_{i j} \sim M_{\mathrm{Pl}}$ the PGB interactions are extremely weak, so that the main consequences of our theory are in the neutrino mass sector. With 3 right-handed neutrinos, the full neutrino mass matrix is $6 \times 6$ and is made of a Dirac mass matrix, $m_{i j}=\lambda_{i j}^{D}\langle h\rangle$, and of a Majorana mass matrix for the right-handed neutrinos, $M_{i j}=\lambda_{i j} f_{i j}$, both $3 \times 3$ and roughly of the same size (up to differences among the various matrix elements, which can of course be significant). An $M$ very much smaller or much bigger than $m$ would in fact give the wrong size for the PGB potential. Such a neutrino mass matrix is mainly constrained by oscillation experiments. However, it is also constrained by cosmology: extra sterile neutrinos coming into equilibrium, partially or totally, affects Big-Bang Nucleosynthesis (BBN), the Cosmic Microwave Background (CMB) and Large Scale Structure (LSS) formation.

After symmetry breaking, the neutrino masses and mixings can be described in full generality by

$$
\mathscr{L}=\frac{g}{\sqrt{2}} \bar{\nu} V \gamma_{\mu} P_{L} e W_{\mu}+e^{T} m_{E} e^{c}+\nu^{T} m^{d} N+\frac{1}{2} N^{T} U^{T} M^{d} U N+\text { h.c. }
$$

where the flavour indices are left understood, $m_{E}, m^{d}$ and $M^{d}$ are real and diagonal matrices, $V$ is a unitary matrix with a single physical phase and $U$ is a unitary matrix with five physical phases ${ }^{2}$. If $m$ and $M$ are diagonalized by $m=V_{D} m^{d} U_{D}$ and $M=U_{M}^{T} M^{d} U_{M}$ respectively, then $U=U_{M} U_{D}^{\dagger}$. To analyze the constraints in full generality in the entire space of parameters is complicated and goes beyond the scope of this work. In the following we try to describe the main features of the allowed parameter space.

We first consider the special case $U=1$, in which $m$ and $M$ may be simultaneously diagonalized. This is a useful starting point to understand the more general situation or, at least, to show that there are allowed regions in parameter space that fulfil all requirements. This situation is fully realistic and we study it below.

The constraints on the mass parameters are easy to determine because diagonalization of the $6 \times 6$ neutrino mass matrix decouples into 3 separate $2 \times 2$ diagonalizations, one for each $\left(\nu_{i}, N_{i}\right)$ pair. We disregard possible degeneracies and order the eigenvalues of the Dirac and Majorana mass matrices, $m_{i}$ and $M_{i}$ respectively, in such a way that $m_{3}, M_{3}$ govern the atmospheric oscillation length and $m_{2}, M_{2}$ the solar oscillation length. The constraints from oscillation experiments on $M_{2}$ and $M_{3}$ are shown in Fig. 1. Also shown are the respective fractions of thermalized sterile neutrinos at $\mathrm{BBN}$ and $\mathrm{CMB}$ eras, $\Delta N_{\nu}$, and the regions excluded by LSS data. An analogous figure cannot be drawn for $M_{1}$ since we do not know the mass of the lightest active, or quasi-active neutrino. However, for $m_{1}$ small enough, say $m_{1} \lesssim 10^{-6} \mathrm{eV}, M_{1}$ is almost unconstrained.

While LSS forbids $M_{2,3} \gtrsim \mathrm{eV}$, each of these masses could lie in the "0.3 eV window", given by $0.1 \mathrm{eV} \lesssim M_{2,3} \lesssim \mathrm{eV}$. Since the observed masses for atmospheric and solar oscillations are less

\footnotetext{
${ }^{2}$ The proof is as follows. The first three terms in the right-hand-side of 8 are the usual terms in the case of pure Dirac neutrinos, which can always be reduced to this form. The last term is an arbitrary symmetric matrix with the overall phase which is unphysical because it can be reabsorbed by an overall lepton number transformation.
} 

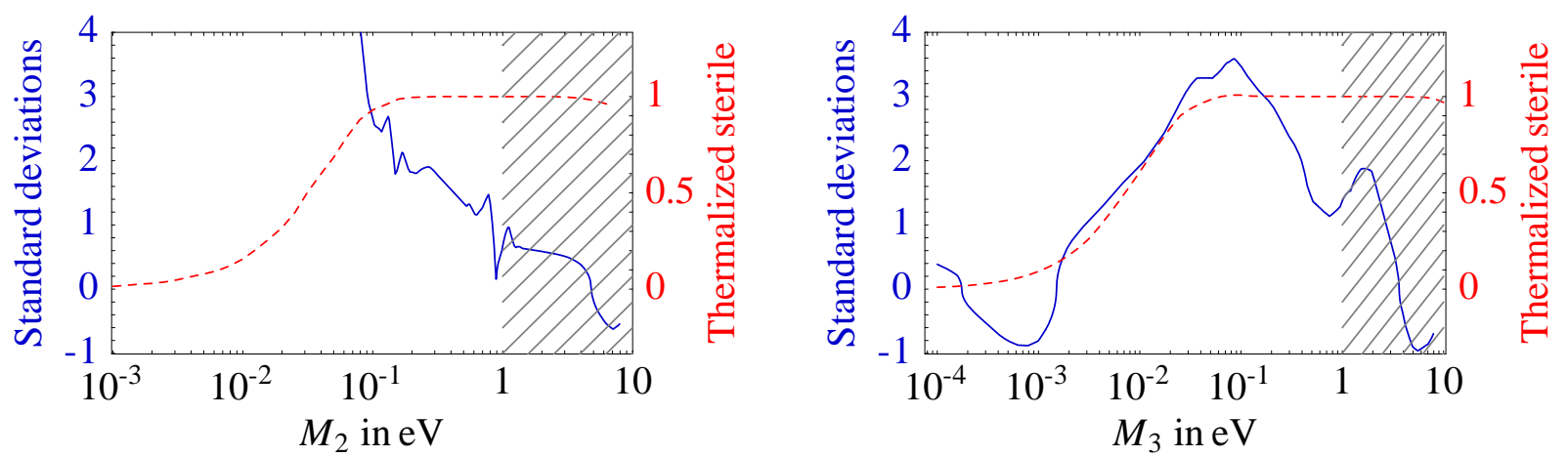

Figure 1: $\operatorname{Sign}\left(\Delta \chi^{2}\right) \cdot\left|\Delta \chi^{2}\right|^{1 / 2}$ of the global oscillation fit (continuous blue line/left vertical axis) and thermalized sterile fraction (red dotted line/right vertical axis). Large Scale Structure data exclude thermalized heavy sterile neutrinos (shaded regions).

than $0.3 \mathrm{eV}$, these values for $M_{2,3}$ lead to a mini-seesaw. Alternatively, although atmospheric oscillations exclude $M_{3} \simeq m_{3}$, any value less than about $10^{-2} \mathrm{eV}$ is allowed, yielding a pseudoDirac pair for $\left(\nu_{3}, N_{3}\right)$. On the other hand, solar oscillations forbid $M_{2}$ beneath the $0.3 \mathrm{eV}$ window all the way down to $\sim 10^{-9} \mathrm{eV}$. Allowed values below $10^{-9} \mathrm{eV}$ lead to a pseudo-Dirac $\left(\nu_{2}, M_{2}\right)$ pair. Thus, each of $\left(\nu_{2}, M_{2}\right)$ and $\left(\nu_{3}, M_{3}\right)$ either undergo a mini-seesaw or form a pseudo-Dirac pair. Furthermore, from Fig. 1 we see that the cosmological thermalization of the sterile state is complete for the mini-seesaw case and absent for the pseudo-Dirac case (except as $M_{3}$ approaches $10^{-2} \mathrm{eV}$, when partial thermalization occurs). Hence we can identify three possibilities ${ }^{3}$

(0) $\Delta N_{\nu} \approx 0: \quad\left(M_{2}, M_{3}\right) \approx\left(10^{-9}, 10^{-3}\right) \mathrm{eV}$

(1) $\Delta N_{\nu} \approx 1: \quad(1 \mathrm{a}) \quad\left(M_{2}, M_{3}\right) \approx\left(0.3,10^{-3}\right) \mathrm{eV}$, or $(1 \mathrm{~b}) \quad\left(M_{2}, M_{3}\right) \approx\left(10^{-9}, 0.3\right) \mathrm{eV}$.

(2) $\Delta N_{\nu} \approx 2: \quad\left(M_{2}, M_{3}\right) \approx(0.3,0.3) \mathrm{eV}$

These four mass ranges correspond to the four possible ways of assigning mini-seesaw and pseudoDirac spectra to each of $\left(\nu_{2}, M_{2}\right)$ and $\left(\nu_{3}, M_{3}\right)$, as shown in Fig. 2. One extra neutrino at BBN looks compatible with standard cosmology, with systematic effects taken into account, whereas two extra neutrinos appear definitely problematic, unless one invokes an ad hoc non standard cosmology, such as large lepton asymmetries or a MeV-scale reheating temperature.

Although we have set $U=1$, the unitary matrix $U_{M}=U_{D}$ is completely undetermined by neutrino mass phenomenology - the Euler angles, $\theta$, of $U_{M}$ can be chosen to obtain the observed DE, $\rho_{\mathrm{DE}}$. If $M_{1}$ is sufficiently small, the relevant entries of $M_{i j}=\lambda_{i j} f_{i j}$ are given by Euler angles

\footnotetext{
${ }^{3}$ Here $0.3 \mathrm{eV}$ means anywhere in the " $0.3 \mathrm{eV}$ window", $M_{3} \approx 10^{-3} \mathrm{eV}$ means any value of $M_{3}$ less than about $10^{-2} \mathrm{eV}$, and $M_{2} \approx 10^{-9} \mathrm{eV}$ means any value of $M_{2}$ less than about $10^{-9} \mathrm{eV}$.
} 


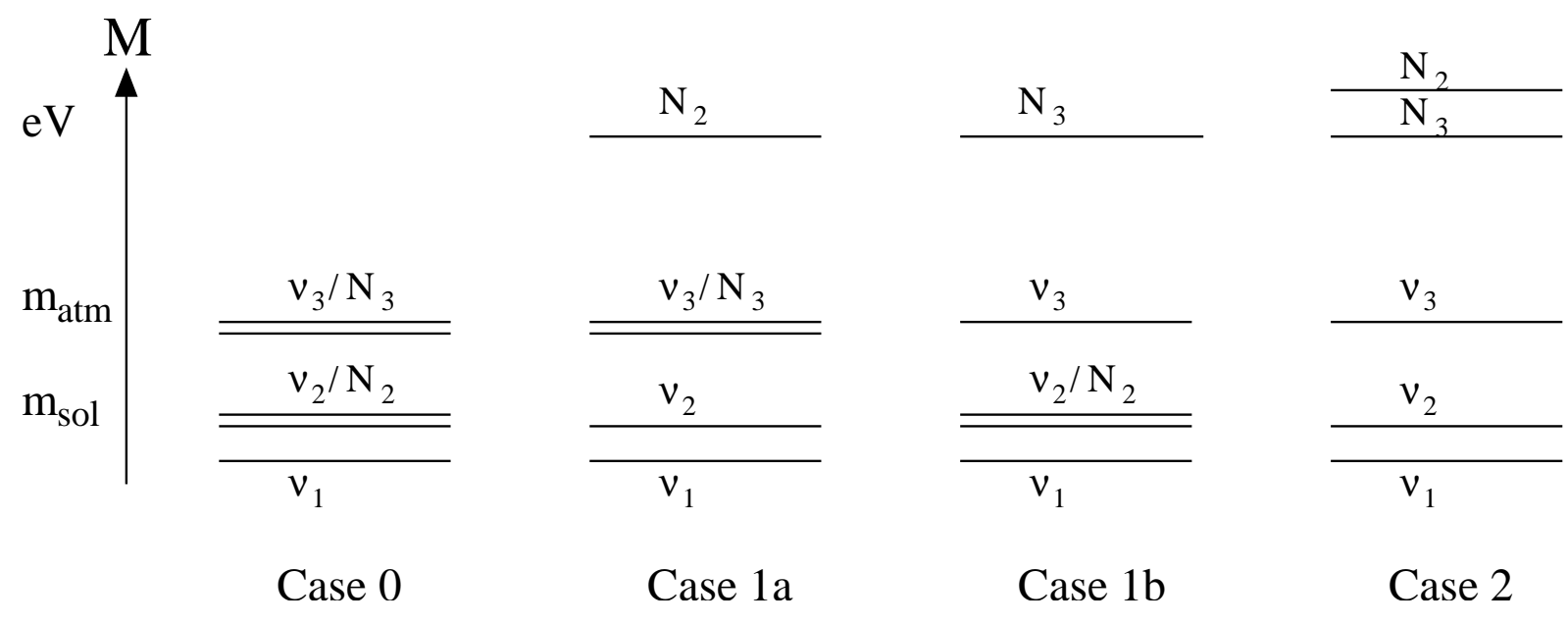

Figure 2: Schematic illustration of four possible neutrino mass spectra consistent with oscillation and cosmological constraints. The mass of $N_{1}$ is largely undetermined.

multiplied by $M_{2}$ or $M_{3}$. In cases (0), (1) and (2), $\rho_{\mathrm{DE}}$ typically requires $\theta \approx 1,10^{-2}$ and $10^{-4}$ respectively. Perhaps the case (0) is most natural, and, depending on the precise value for $M_{3}$, could lead to an observable deviation of $\Delta N_{\nu}$ from 0 .

Theories with $U \neq 1$ can be constructed that are both more natural and more predictive than the $U=1$ case. In particular, suppose that, in the original basis for $N$, we have the texture

$$
M_{i j}=\lambda_{i j} f_{i j}=\left(\begin{array}{ccc}
M_{1} & \varepsilon_{12} & \varepsilon_{13} \\
\varepsilon_{12} & M_{2} & \varepsilon_{23} \\
\varepsilon_{13} & \varepsilon_{23} & M_{3}
\end{array}\right), \quad m=\left(\begin{array}{ccc}
m_{1} & 0 & 0 \\
0 & m_{2} & 0 \\
0 & 0 & m_{3}
\end{array}\right) \text {. }
$$

where the $\varepsilon_{i j}$ are either zero or sufficiently small that the neutrino phenomenology is not significantly altered from the $U=1$ case. The standard neutrino mixing angles arise from transformations on the left-handed leptons, charged or neutral. In each of the three cases described above, it is possible to introduce a single off-diagonal entry, $\varepsilon_{i j}$, such that the appropriate PGB potential is generated, $\rho_{\mathrm{DE}} \approx M_{i i} M_{i j}^{*} M_{j j} M_{j i}^{*}$ for some $(i, j)$.

Realistic examples, corresponding to cases (0) and (1) above, are

(0) $\left(m_{1}, m_{2}, m_{3}\right) \approx\left(\lesssim 10^{-6} \mathrm{eV}, m_{\mathrm{sun}}, m_{\mathrm{atm}}\right)$ $\left(M_{1}, M_{2}, M_{3}\right) \approx\left(\gtrsim 10^{-3}, 10^{-9}, 10^{-3}\right) \mathrm{eV}$, and $\varepsilon_{13} \approx 10^{-2} \mathrm{eV}$;

(1) $\left(m_{1}, m_{2}, m_{3}\right) \approx\left(0,5 \times 10^{-3} \mathrm{eV}, m_{\mathrm{atm}}\right)$, $\left(M_{1}, M_{2}, M_{3}\right) \approx\left(0,0.3,10^{-8}-10^{-3}\right) \mathrm{eV}$, and $\varepsilon_{23} \approx 10^{-4} \mathrm{eV} \cdot\left(10^{-3} \mathrm{eV} / M_{3}\right)^{1 / 2}$

with other $\varepsilon_{i j}$ taken irrelevantly small. 


\section{Signals and Conclusions}

In promoting a $\mathrm{CP}$ violating phase of the neutrino mass matrix to the DE quintessence field, we are led, essentially uniquely, to a theory with light right-handed neutrinos, with possible spectra shown in Figure 2. This proposal can be tested in neutrino physics.

- The three cases with differing $\Delta N_{\nu}$ will easily be distinguished by precision CMB measurements at PLANCK, and perhaps at WMAP.

- The range $M_{2} \sim 0.3 \mathrm{eV}$ can be completely tested by cosmology (searching for sterile neutrino masses) and by reactor experiments (searching for $\bar{\nu}_{e}$ disappearance at base-line $\sim 10 \mathrm{~m}$ ).

- Similarly, $M_{3} \sim 0.3 \mathrm{eV}$ can be tested by cosmology, and possibly by atmospheric neutrino experiments (HyperK, MONOLITH, IceCube) and beam experiments (MiniBoone, MINOS).

- Long-baseline experiments will probe $M_{3} \sim 10^{-3} \mathrm{eV}$. If $M_{3}$ approaches $10^{-2} \mathrm{eV}$, it can be determined by a CMB or BBN measurement of $\Delta N_{\nu}$, and signals may appear in atmospheric oscillations.

- Very small $M_{1,2,3}$ can give MSW resonances in the sun and in supernovæ, as well as vacuum oscillations of neutrinos that travel cosmological distances.

- MiniBoone is currently testing the LSND anomaly. Constraints from other oscillation data disfavor its interpretation in terms of sterile neutrinos, but do not fully exclude it.

- The detection of a $0 \nu 2 \beta$ signal would exclude this model, since the left-handed neutrinos do not have a direct Majorana mass and the right-handed neutrinos are light, so that effects in $0 \nu 2 \beta$ are suppressed by powers of $M / Q$ (where $Q \sim \mathrm{MeV}$ is the energy released in $0 \nu 2 \beta$ ).

The Mass Varying Neutrino scheme for DE [7] also involves a light scalar coupled to neutrinos. The neutrino energy density plays a crucial role in the dynamics of DE because the scalar is both light and has a significant coupling to the neutrinos. Thus the scheme predicts a characteristic shift in the position of the CMB peaks, corresponding to at least one species of neutrino scattering during the eV era [10]. On the other hand, in our scheme the coupling of neutrinos to light PGBs is proportional to $M / f$, and is so small that all neutrinos free-stream during the CMB era.

We conclude by noting other areas that are worth investigating. Since there are several PGBs, some might have masses larger than todays Hubble parameter, so that they oscillate during the recent evolution of the universe with characteristic signals related to the associated Jeans length [11, 12. Could such a PGB give all of the dark matter? The mass should be larger than about $10^{-22} \mathrm{eV}$, otherwise the uncertainty principle prevents the formation of structures at sufficiently small scales [12. Parameters exist that allow a unified picture of both dark matter and DE. In theories with sufficiently sparse textures, it may be possible to compute the magnitude of the DE and dark matter energy densities from measurements of neutrino masses and mixings. Finally, the (super-)potential that gives the $\phi_{i j}$ a vev at a large scale could play a role in inflation: 
a candidate for a such superpotential is $W=\sum_{i j} S_{i j}\left(\sigma_{i j} \phi_{i j} \bar{\phi}_{i j}-\mu_{i j}^{2}\right)$ where $S_{i j}, \phi_{i j}$ and $\bar{\phi}_{i j}$ are chiral supermultiplets.

\section{Acknowledgments}

We thank D. Larson and Y. Nomura for many conversations. This work is supported in part by MIUR and by the EU under RTN contract MRTN-CT-2004-503369, by DOE under contracts DE-FG02-90ER40542 and DE-AC03-76SF00098 and by NSF grant PHY-0098840.

\section{References}

[1] S. Perlmutter et al. [Supernova Cosmology Project Collaboration], Astrophys. J. 517, 565 (1999) astro-ph/9812133; A. G. Riess et al. [Supernova Search Team Collaboration], Astron. J. 116, 1009 (1998) astro-ph/9805201; A. G. Riess et al. [Supernova Search Team Collaboration], Astrophys. J. 607, 665 (2004) astro-ph/0402512.

[2] For a review see P. J. E. Peebles and B. Ratra, Rev. Mod. Phys. 75, 559 (2003) astro-ph/0207347.

[3] S. Weinberg, Phys. Rev. Lett. 59, 2607 (1987); H. Martel, P. R. Shapiro and S. Weinberg, Astrophys. J. 492, 29 (1998) astro-ph/9701099.

[4] P. J. E. Peebles and B. Ratra, Astrophys. J. 325, L17 (1988); B. Ratra and P. J. E. Peebles, Phys. Rev. D 37, 3406 (1988); C. Wetterich, Nucl. Phys. B 302, 668 (1988).

[5] N. Weiss, Phys. Lett. B 197, 42 (1987).

[6] J. A. Frieman, C. T. Hill, A. Stebbins and I. Waga, Phys. Rev. Lett. 75, 2077 (1995).

[7] R. Fardon, A.E. Nelson and N. Weiner, JCAP 0410, 005 (2004) astro-ph/0309800.

[8] J.E. Kim, JHEP 9905, 022 (1999) hep-ph/9811509; JHEP 0006, 016 (2000) hep-ph/9907528; K. Choi, Phys. Rev. D 62, 043509 (2000) hep-ph/9902292; Y. Nomura, T. Watari and T. Yanagida, Phys. Lett. B 484, 103 (2000); hep-ph/0004182 . J. E. Kim and H.P. Nilles, Phys. Lett. B 553, 1 (2003) hep-ph/0210402; L.J. Hall, Y. Nomura, S. J. Oliver, astro-ph/0503706.

[9] C.T. Hill and G. G. Ross, Nucl. Phys. B 311, 253 (1988).

[10] Z. Chacko, L.J. Hall, T. Okui, and S. Oliver Phys. Rev. D 70, (2004) 085008 hep-ph/0312267.

[11] M. Khlopov, B. Malomed and Ya. B. Zel'dovich, Mon. Not. R. Astron. Soc. 215, 575 (1985).

[12] W. Hu, R. Barkana and A. Gruzinov, Phys. Rev. Lett. 85, 1158 (2000) astro-ph/0003365. 